\title{
Dynamics of Flexoelectric Materials: Subsonic, Intersonic, and Supersonic Ruptures and Mach Cone Formation
}

\author{
Antonios E. Giannakopoulos \\ Mechanics Division, \\ National Technical University of Athens, \\ Zografou 15773, Greece \\ e-mail: agiannak@uth.gr \\ Ares J. Rosakis \\ Graduate Aerospace Laboratory, \\ California Institute of Technology, \\ Pasadena, CA 91125 \\ e-mail: arosakis@caltech.edu
}

Motivated by recent, unexpected, experimental observations of "intersonic" rupture growth in which both shear and dilatational Mach fronts were observed at the tips of dynamic frictional ruptures propagating at rupture speeds below the dilatational wave speed of the surrounding solid, and we formulate the general dynamic flexoelectric problem and we investigate its plane strain/plane polarization specialization. The coupling of the mechanical problem is analogous to a problem of Toupin-Mindlin gradient elasticity, where two micromechanical characteristic lengths and two microinertial lengths emerge as a combination of the mechanical, dielectric, and flexoelectric constants. The solution of the rupture growth problem allows us to provide an explanation of the experimental results. This becomes possible since flexoelectricity predicts a new aspect that was not observed in the classical analysis: subsonic super shear and supersonic crack tip (or rupture) motions are not related exclusively with the problem being elliptic or hyperbolic, respectively. This is due to the influence of the microinertial lengths, which, in addition to the ratios of the rupture to the wave speeds, also affect the slopes of the Mach cones. Moreover, we are able to explain the experimental paradox of the observation of double Mach cone pairs at the tips of supershear, but subsonic, frictional, ruptures in poly-methyl-methacrtylate (PMMA) by demonstrating that both dilatational and shear Mach cones could appear in flexoelectric solids at rupture speeds below the material dilatation wave speed, something that is impossible from the classical elasticity analysis and is due to the dispersive nature of the present problem. Our analysis is of relevance to the dynamic deformation and fracture of both synthetic and naturally occurring flexoelectric materials and systems, with implications to both engineering and earthquake source mechanics. [DOI: 10.1115/1.4046634]

Keywords: flexoelectricity, dynamic elasticity, crack/rupture propagation, Mach shock fronts, length scales, ellipticity/hyperbolicity, constitutive modeling of materials, micromechanics

\section{Introduction}

Flexoelectricity is the ability of materials to convert mechanical strain gradients to electric polarization and vice versa. This implies that dielectric materials and ferroelectrics in their paraelectric phase, under inhomogeneous mechanical strain, can produce polarization, and so they can classify as flexoelectrics (see for example, Refs. [1-3]). Many rocks that exist within the earth's crust and mantle exhibit flexoelectricity, often combined with piezoelectricity (in case of anisotropy). Excellent recent perspective of this unusual electromechanical coupling with emphasis on applications to energy harvesting, microelectromechanical systems, nanotechnology, and biology can be found in Ref. [4] as well as in other review articles such as in Refs. [5,6], to mention but a few. Materials in which flexoelectricity has been observed include ribbons of graphene, boron nitride and carbon nitride, biological membranes, and polymers such as plexiglass (poly-methyl-methacrylate (PMMA)), paraffin, and polystyrene (see, for example, Refs. [7-9]). Of particular interest in this work is crystalline materials such as salt, strodium titanate, barium titanate, ice, sulfur, red phosphorous, cesium iodide, semiconductors, and many more complex oxide ceramics such as peroviskites [6,10-12], alkali halides [13], and magnesium oxide [14]. Although the focus is on the flexoelectric behavior, we should mention that the general

Contributed by the Applied Mechanics Division of ASME for publication in the Journal of APPLIED MeChanics. Manuscript received February 28, 2020; final manuscript received March 2, 2020; published online March 11, 2020. Assoc. Editor: Yonggang Huang. influence of the electron density distribution even for centrosymmetric materials with metallic bonding was indicated early on by Weiner [15]. Weiner showed that unless the electron density distribution deforms according to affine transformations, the Cauchy symmetries that characterize classical elasticity could be violated giving nonsymmetric stress tensors. The dielectric loss in polymers and ionic crystals, at moderate temperatures, can be correlated with the internal friction and is associated with some side molecular or ionic motion ( $\beta$-relaxation). Therefore, in these materials, mechanical visoelasticity has been shown to be analogous to the dielectric relaxation, see, for example, Ref. [16].

Several models of flexoelectricity have been proposed so far ranging from atomistic $[17,18]$ to continuum approaches $[19,20]$ followed by a vast number of approximate analytic and numerical solutions. However, many of the proposed solutions are approximate in the sense that they neglect the inverse flexoelectric effect, often tagged as pseudopiezoelectric or as surface-piezoelectric solutions. This, in effect, means that the gradient of polarization must be included in the formulation, as was suggested by Mindlin [21]. The first systematic attempt in this direction can be found in the work of Sahin and Dost [22].

Turning attention on dynamic flexoelectricity, only limited amount of results can be found-see, for example, Refs. [22,23]. We would like to note at this point the lack of material data related to flexoelectricity and to emphasize the possible experimental problems that might stem from nonincluding the inverse flexoelectric effect in the analysis.

The flexoelectric effect is in general weaker than the piezoelectric effect and attempts to enhance it in manufactured solids by tailoring 
their microstructure can be found in several works that include composites or specific shapes of porosity (see Ref. [4] and references therein). At small scales, however, the flexoelectric effect is indeed important, which makes it paramount in the vicinity of defects in all dielectrics due to the strong strain inhomogeneities encountered, for example, at crack tips and dislocation cores. A first attempt to model such defects can be found in the work of Mao and Purohit [24] who presented a static analysis of cracks and dislocations by introducing a strain gradient elasticity methodology, however, neglecting the inverse flexoelectric effect (i.e., the polarization gradient).

Regarding the importance of dynamics, it should be noted that there are many practical cases where stress wave- and shock wave-induced loading enhances the flexoelectric response and influences materials and device reliability. As only one example, the protection of electric devises and high-pressure diagnostic equipment from spurious electrical effects, when mechanically shocked under operation (e.g., impact from accidental drop, explosion), brings about the dramatic drop of electric resistivity of many dielectrics subjected to mechanical shock conditions. One can see, for example, the early works of Brich et al. and Joigneau and Thaivervin $[25,26]$, where it was found experimentally that electric voltage appeared due to mechanically induced polarization, proportional to the shock pressure, corresponding to the entrance and exit of the shock fronts. At the shock front, the strain gradient is very high; therefore, the flexoelectric phenomenon could account for such electric resistivity observations. At a vastly different length scale, such phenomena are also to be expected in Seismology and Earthquake Source Mechanics [27], as dynamic shear ruptures propagating along faults are long known to be the key mechanism of generating earthquakes [28].

The recent experimental discovery of super shear dynamic ruptures, whose speeds exceed the shear wave speed [28-33], has once again brought to the forefront the connection between shock wave fronts and the flexoelectric response and has inspired the present study. Indeed, such shear ruptures, like frictional earthquake ruptures, propagate along week planes and have been shown to be able to exceed characteristic material wave speeds. The laboratory experiments, typically conducted in specimens made of polymers such as Homalite-100, have revealed the existence of Mach line structures originating at their dynamically moving rupture tips as expected by classical elasticity when characteristic wave speeds are exceeded by the ruptures. Such structures, which originate at the dynamically moving rupture tips, propagate almost unattenuated away from the fracture or the frictional fault planes, while sweeping the solid, and feature very large strain rates along their length and very high local strain gradients across them [33]. As we discuss in the present paper, these gradients are expected to greatly enchase the ferroelectric effect and to produce local phenomena observable by detailed and dynamic experimental measurements of mechanical field quantities and their gradients near the growing rupture fronts. Regarding "real earthquakes," the experimental discovery of supershear ruptures in the lab has motivated field seismologist to look closer at field evidence for large-magnitude earthquakes propagating at the supershear speed, and as a result, the reporting of such events, formerly thought to be rare, have significantly multiplied (e.g., Refs. [34-38]). Given that supershear rupture is indeed a possibility during large real earthquakes and that many crustal rock types (much like some of the polymers used in the experiments) are also thought to be flexoelectric, the existence of the anticipated Mach lines at the rupture tips is expected to promote especially strong flexoelectric effects during supershear natural earthquakes and are thus of great relevance to the present study.

Indeed, the most recent dynamic frictional shear rupture experiments by Gori et al. [39] have provided yet the strongest motivation for the present analysis. Some of the experiments reported in that study was different than our earlier ones in two respects. First, they were conducted using specimens of PMMA. Second, they used a new, ultra-fast, diagnostic technique $[40,41,33]$ capable of recording full-field maps of all components of displacement, strain, and strain rate near the propagating rupture in real time. This method, called dynamic digital image correlation (DIC), has been used in conjunction with ultra-high-speed photography to accurately visualize strain discontinuities (such as those associated with Mach lines), more accurately than ever before [33]. The experiments of Gori et al. revealed ruptures propagating at speeds way above the dynamic shear wave speed but just below the dynamic dilatational wave speed of PMMA. According to the classical elasticity theory, this would imply the existence of only one set of Mach lines (shear strain discontinuities) emitted from the rupture tip since only the shear wave speed was exceeded. Instead, the measurement revealed two distinct sets of Mach lines (one featuring shear and the other dilatational strain jumps). This is an observation, which is clearly inconsistent with classical elasticity predictions, and its explanation necessitates invoking a more complex constitutive description of the solid hosting the rupture. This description could be, for example, a flexoelectric constitutive law of the type used in the analysis of moving screw dislocations that has recently been considered by Giannakopoulos and Th [42] or a viscoelastic constitutive description as discussed and proposed by Gori et al. Giannakopoulos and $\mathrm{Th}$ have concentrated on the antiplane problem, i.e., the screw dislocation problem moving with the constant speed. Their analysis showed similar interplay of the dynamics of the screw dislocation motion with the general shear waves that will be treated in this work: possible appearance of shear Mach lines under rupture speeds that are less than the characteristic shear wave speed.

Motivated by the above experimental observations, we first pose here the general 3D dynamic problem of dynamic, flexoelectricity, and also specialize it in two dimensions. We then investigate the in-plane, steady-state ruptures in flexoelectric solids and explore the unusual Mach line phenomena that occur as the rupture tip speeds are increased and exceed, characteristic wave speeds of the solid. The starting point for explaining the experimental observations is solving the dynamic in-plane crack problem of an elastic dielectric solid that includes the effect of polarization gradient and flexoelectricity due to strain gradient. Here, flexoelectricity is considered to be the only source of strain gradient effects since most amorphous materials (such as PMMA) have very small purely elastic nonlocal lengths [43]. We show that the coupling of the mechanical problem is analogous to a problem of Toupin-Mindlin gradient elasticity where two micromechanical characteristic lengths and two microinertial length emerge as a combination of the mechanical, dielectric, and flexoelectric constants. The microstructural lengths connect to the (well known in the context of gradient elasticity) displacement curvature. The microinertial lengths are less referenced (and hardly considered in metrology) essentially introduce a nonclassic kinetic energy term that connects to the microrotations of the matter. Moreover, the nonclassical boundary conditions related to the dynamic gradient elasticity are naturally related to the dielectric boundary conditions. As a result, regarding the mechanical response, the dynamic flexoelectric problem reduces to a dynamic gradient elasticity problem.

The solution of the reduced, in-plane, rupture growth problem allows us to directly connect with the unexpected experimental observations of dual (shear and dilatational Mach lines) for supershear but still subsonic rupture growth. This becomes possible since flexoelectricity predicts a new aspect that was not observed in the classic analysis: subsonic supershear and supersonic rupture tip motions are not related exclusively with the problem being elliptic or hyperbolic, respectively. This has to do with the influence of the emerging microinertial lengths, which also affect the slope of the Mach cones. Moreover, dilatational Mach cones could appear at rupture speeds below the material dilatation wave speed, something that is impossible from the classical elasticity analysis and is due to the dispersive nature of present problem.

\section{Continuum Flexoelectricity}

We examine a homogeneous linear flexoelectric solid (being dielectric at the same time) with an energy density due to elastic 
deformation and electric polarization, which depends on the strain gradients. Reverse flexoelectricity implies that the gradient of the polarization produces strain and should be included in the energy density. The elastic strain energy due to strain gradient effects will not be considered, and the kinetic strain energy will not include microrotational effects. These assumptions mean that there are no a priori internal length scales as implied in nonlocal elastic theories. However, we will show that the coupling of the mechanical and the electrical problem gives rise to microstructural and microinertial lengths resulting from the coupling.

In what follows, consider the flexoelectric problem with key unknowns, the material displacement vector $u_{i}(\mathrm{~m})$, the material polarization vector $P_{i}\left(\mathrm{C} / \mathrm{m}^{2}\right)$, and the electric potential $\Phi(\mathrm{Nm} /$ C). These are functions of the (right-handed) Cartesian coordinates $x_{1}, x_{2}$, and $x_{3}$ and the time $t$. The linear internal energy density function that includes deformation and polarization is given as follows $[19,21,23]$ :

$$
W=\left[\begin{array}{c}
\frac{1}{2} a_{i j} P_{i} P_{j}+\frac{1}{2} b_{i j k l} P_{j, i} P_{l, k}+\frac{1}{2} c_{i j k l} \varepsilon_{i j} \varepsilon_{k l}+e_{i j k l} P_{j, i} \varepsilon_{k l} \\
+f_{i j k l} P_{i} \varepsilon_{k l, j}+b_{i j}^{0} P_{j, i}
\end{array}\right]
$$

The mechanical linear strain is related to the displacement vector as $\varepsilon_{i j}=\left(u_{i, j}+u_{j, i}\right) / 2 . P_{i, j}$ is the gradient of the polarization vector $P_{i}$, and $\varepsilon_{i j, k}$ are the gradients of the strains. Repeated indices imply summation from 1 to 3 and ()$_{, i}=\partial / \partial x_{i}$. The compatibility equations are identical to classic linear elasticity. The form of the energy density function (1) omits an extra term that ensures thermodynamic stability of the total energy $\left(1 / 2 g_{i j k l m n} u_{i, j k} u_{l, m n}\right)$. This term represents the contribution of purely elastic nonlocal effects.

It has been found however (see erratum in Ref. [19]) that, for most problems, excluding this contribution is generally small, although, if flexoelectricity is incorporated, it is required to guarantee thermodynamic stability. For some problems, this omission (or inclusion) of this term may be important especially where the stability is an issue.

The material constants are as follows: the elastic constant tensor $c_{i j k l}\left(\mathrm{~N} / \mathrm{m}^{2}\right)$, the flexoelectric coefficient tensor $f_{i j k l}(\mathrm{Nm} / \mathrm{C})$, the reciprocal dielectric susceptibility tensor $a_{i j}\left(\mathrm{Nm}^{2} / \mathrm{C}^{2}\right)$, the inverse flexoelectric coefficient tensor $e_{i j k l}(\mathrm{Nm} / \mathrm{C})$, and the gradient polarization coupling tensor $b_{i j k l}\left(\mathrm{Nm}^{4} / \mathrm{C}^{2}\right)$. The symmetries of the above constants have been addressed in Ref. [44]. The constants $b_{i j}^{0}$ are related to the surface energy per unit area $T_{s}=\left(n_{i} b_{i j}^{0} P_{j}\right) / 2$ with $n_{i}$ being the unit normal vector pointing outside the flexoelectric body [21].

The Maxwell electric self-field is $E_{i}=-\Phi_{, i}(\mathrm{~N} / \mathrm{C})$. The total electric enthalpy is as follows:

$$
\bar{H}=W-\frac{1}{2} \varepsilon_{0} \Phi_{, i} \Phi_{, i}+\Phi_{, i} P_{, i}
$$

where $\varepsilon_{0} \approx 8.854 \times 10^{-12} \mathrm{C}^{2} / \mathrm{N} / \mathrm{m}^{2}$ is the dielectric permittivity of vacuum (assumed to surround the body) [45].

The kinetic energy density is as follows:

$$
T=\frac{1}{2} \rho \dot{u}_{3} \dot{u}_{3}
$$

where $\rho$ is the material mass density and $\dot{u}_{i}=\partial u_{i} / \partial t$ is the material velocity vector. If $\rho=0$, the problem reduces to the static case.

Accordingly, the constitutive equations are written as follows:

(1) Cauchy (symmetric) stress tensor:

$$
\sigma_{i j}=\frac{\partial W}{\partial \varepsilon_{i j}}=c_{i j k l} \varepsilon_{k l}+e_{k l i j} P_{l, k}
$$

(2) Dipolar stress tensor:

$$
\tau_{i j k}=\frac{\partial W}{\partial \varepsilon_{j k, i}}=f_{l i j k} P_{l}
$$

(3) Effective local electric force:

$$
\bar{E}_{k}=-\frac{\partial U}{\partial P_{k}}=-\left(a_{k j} P_{j}+f_{k l i j} \varepsilon_{i j, l}\right)
$$

(4) Polarization gradient force:

$$
E_{i j}=\frac{\partial W}{\partial P_{j, i}}=b_{i j k l} P_{l, k}+e_{i j k l} \varepsilon_{k l}+b_{i j}^{0}
$$

We will concentrate in the isotropic response [23], and in this case, the material tensors become

$$
\begin{gathered}
a_{i j}=a \delta_{i j} \\
c_{i j k l}=c_{12} \delta_{i j} \delta_{k l}+c_{44}\left(\delta_{i k} \delta_{j l}+\delta_{j k} \delta_{i l}\right) \\
f_{i j k l}=f_{12} \delta_{i j} \delta_{k l}+f_{44}\left(\delta_{i k} \delta_{j l}+\delta_{j k} \delta_{i l}\right) \\
e_{i j k l}=e_{12} \delta_{i j} \delta_{k l}+e_{44}\left(\delta_{i k} \delta_{j l}+\delta_{j k} \delta_{i l}\right) \\
b_{i j k l}=b_{12} \delta_{i j} \delta_{k l}+b_{44}\left(\delta_{i k} \delta_{j l}+\delta_{j k} \delta_{i l}\right)+b_{77}\left(\delta_{i k} \delta_{j l}-\delta_{j k} \delta_{i l}\right) \\
b_{i j}^{0}=b_{0} \delta_{i j}
\end{gathered}
$$

where $\delta_{i j}=0$ is Kronecker's delta (identity tensor). All material constants are positive definite and bounded. The dielectric susceptibility $\chi$ relates to the dielectric constant of vacuum $\varepsilon_{0}$ as $1 / a=\chi \varepsilon_{0}$. The classic elastic dielectric case is obtained if $f_{i j k l}=0$ and $e_{i j k l}=0$, whereas the classic elastic case requires additionally $a_{i j}=0$ and $b_{i j}^{0}=0$. If only $f_{i j k l}=0$, we recover the formulation of Mindlin $[21,46]$ for a dielectric solid with polarization gradient.

Using Hamilton's principle (least action), which is minimization of the total electric enthalpy with respect to $u_{i}$ and $P_{i}$ in the whole body volume $V$ and arbitrary time interval $0, t_{1}$,

$$
\int_{0}^{t_{2}} \int_{V} \delta(\bar{H}-T) d V d t=0
$$

we obtain the Euler conditions for all the material points of the body (in the presence of body forces $X_{i}\left(\mathrm{~N} / \mathrm{m}^{3}\right)$ and initial electric field $E_{i}^{0}$ (N/C):

(1) Conservation of linear momentum:

$$
\sigma_{j i, j}-\tau_{k j i, j k}+X_{i}=\rho \ddot{u}_{i}
$$

(2) Conservation of electric field:

$$
\bar{E}_{j}+E_{i j, i}-\Phi_{, j}+E_{j}^{0}=0
$$

(3) Maxwell equations inside the body:

$$
-\varepsilon_{0} \Phi_{, i i}+P_{i, i}=0
$$

(4) Maxwell equations outside the body become:

$$
\nabla^{2} \Phi=\Phi_{, 11}+\Phi_{, 22}+\Phi_{, 33}=0
$$

The corresponding, work conjugate, boundary conditions are summarized in Table 1 . The electric boundary conditions can be materialized with appropriate steady-state currents applied by surface conductors [47]. 
Table 1 Mutually exclusive boundary conditions for the flexoelectric problem

Essential boundary

conditions

Dynamic boundary conditions

\begin{tabular}{ll}
\hline$P_{i}$ & $n_{i} E_{i j}$ \\
$\Phi$ & $n_{i}\left(-\varepsilon_{0} \llbracket \Phi_{, i} \rrbracket+P_{i}\right)=0$ \\
$D u_{i}$ & $r_{i}=\tau_{k j i} n_{k} n_{j}$ \\
$u_{i}$ & $t_{i}=\sigma_{i j} n_{j}-\tau_{k j i, k} n_{j}+\left(D_{l} n_{l}\right) n_{j} n_{k} \tau_{k j i}-D_{j}\left(\tau_{k j i} n_{k}\right)$
\end{tabular}

Notes: $n_{i}$ is the unit normal vector pointing outside the body, $D \equiv n_{k} \partial / \partial x_{k}$ is the normal to the surface derivative, $D_{j} \equiv\left(\delta_{j k}-n_{j} n_{k}\right) \partial / \partial x_{k}$ is the tangential to the surface derivative, and $\mathbb{I} \rrbracket=()^{+}-()^{-}$is the jump from outside of the body (+) to the inside of the body (-).

The initial conditions are as follows:

$$
\begin{aligned}
u_{i}(\mathbf{x}, 0) & =u_{i}^{0}(\mathbf{x}) \\
\dot{u}_{i}(\mathbf{x}, 0) & =\dot{u}_{i}^{0}(\mathbf{x}) \\
P_{i}(\mathbf{x}, 0) & =P_{i}^{0}(\mathbf{x})
\end{aligned}
$$

where $u_{i}^{0}$ is the initial displacement vector, $\dot{u}_{i}^{0}$ is the initial velocity vector, and $P_{i}^{0}$ is the initial polarization vector. The initial fields are considered to be known and are often taken to be zero.

Furthermore, assuming zero body forces and initial electric field $\left(X_{i}=0, E_{i}^{0}=0\right)$, we transform Eqs. (15) and (16) into Navier-type of equations:

$$
\begin{aligned}
c_{44} \nabla^{2} u_{i} & +\left(c_{12}+c_{44}\right) \nabla_{i}\left(\nabla_{k} u_{k}\right)+\left(e_{44}-f_{12}\right) \nabla^{2} P_{i} \\
& +\left(e_{12}+e_{44}-2 f_{44}\right) \nabla_{i}\left(\nabla_{k} P\right)_{k}=\rho \ddot{u}_{i}
\end{aligned}
$$

$$
\begin{gathered}
\left(e_{44}-f_{12}\right) \nabla^{2} u_{i}+\left(d_{12}+d_{44}-2 f_{44}\right) \nabla_{i}\left(\nabla_{k} u_{k}\right)+\left(b_{44}+b_{77}\right) \nabla^{2} P_{i} \\
+\left(b_{12}+b_{44}-b_{77}\right) \nabla_{i}\left(\nabla_{k} P\right)_{k}-a P_{i}-\Phi_{, i}=0
\end{gathered}
$$

where $\nabla^{2}=\nabla_{k} \nabla_{k}=\partial^{2} / \partial x_{1}^{2}+\partial^{2} / \partial x_{2}^{2}+\partial^{2} / \partial x_{3}^{2}$ is the Laplacian operator and $\nabla^{4}=\nabla^{2} \nabla^{2}$ is the biharmonic operator. Note that, if $f_{i j k l}=0, e_{i j k l}=0$, and $a_{i j}=0$, we obtain from Eq. (20) the classic elastodynamic equations and Eq. (21) is identically zero.

We now focus in the general solution of Eqs. (20) and (21) together with Eq. (17). In the Appendix, we show that the complete solution can be formulated as a Helmholtz decomposition of both the displacement and the polarization vectors as follows:

$$
\begin{array}{ll}
\mathbf{u}=\nabla \phi+\nabla \times \mathbf{H}, & \nabla \cdot \mathbf{H}=0 \\
\mathbf{P}=\nabla \chi+\nabla \times \mathbf{K}, & \nabla \cdot \mathbf{K}=0
\end{array}
$$

where $\nabla$ is the gradient operator, $\phi(\mathbf{x}, t)$ and $\chi(\mathbf{x}, t)$ are scalar functions, whereas $\mathbf{H}(\mathbf{x}, t)$ and $\mathbf{K}(\mathbf{x}, t)$ are vector functions that are solutions of

$$
\begin{gathered}
\nabla^{2} \phi-\ell_{p}^{2} \nabla^{4} \phi=\frac{1}{c_{p}^{2}}\left(\ddot{\phi}-h_{p}^{2} \nabla^{2} \ddot{\phi}\right) \\
\nabla^{2} \mathbf{H}-\ell_{s}^{2} \nabla^{4} \mathbf{H}=\frac{1}{c_{s}^{2}}\left(\ddot{\mathbf{H}}-h_{s}^{2} \nabla^{2} \ddot{\mathbf{H}}\right) \\
\nabla^{2} \chi-\ell_{p}^{2} \nabla^{4} \chi=\frac{1}{c_{p}^{2}}\left(\frac{e_{11}-f_{11}}{a+\varepsilon_{0}^{-1}} \nabla^{2} \ddot{\phi}\right) \\
\nabla^{2} \mathbf{K}-\ell_{s}^{2} \nabla^{4} \mathbf{K}=\frac{1}{c_{s}^{2}}\left(\frac{e_{44}-f_{44}}{a} \nabla^{2} \ddot{\mathbf{H}}\right)
\end{gathered}
$$

where the characteristic dilatation and shear speeds appear as in the classic elastodynamics

$$
\begin{aligned}
& c_{p}=\sqrt{\frac{c_{11}}{\rho}}=\sqrt{\frac{c_{12}+2 c_{44}}{\rho}}=\sqrt{\frac{\lambda+2 \mu}{\rho}}=\sqrt{\frac{E(1-\nu)}{\rho(1+\nu)(1-2 \nu)}} \\
& c_{s}=\sqrt{\frac{c_{44}}{\rho}}=\sqrt{\frac{\mu}{\rho}}=\sqrt{\frac{E}{2 \rho(1+\nu)}}<c_{p}
\end{aligned}
$$

where $E$ and $\nu$ are the Young's modulus and the Poisson's ratio, respectively, and $(\lambda, \mu)$ are the Lame constants. Moreover, in the above equations, four lengths appear, defined by

$$
\begin{gathered}
\left\{\mu, a, f_{12}, f_{44}, e_{44}, b_{44}+b_{77}, \mu\left(b_{44}+b_{77}\right)-e_{44}^{2}\right\}>0 \\
\ell_{s}^{2}=\frac{b_{44}+b_{77}}{a}-\frac{\left(e_{44}-f_{12}\right)^{2}}{\mu a} \geq 0 \\
h_{s}^{2}=\frac{\left(b_{44}+b_{77}\right)}{a} \geq \ell_{s}^{2} \geq 0 \\
\left\{b_{11}=b_{12}+2 b_{44}, a, f_{11}=f_{12}+2 f_{44}, f_{44},(\lambda+2 \mu) b_{11}-e_{11}^{2}\right\}>0 \\
\ell_{p}^{2}=\frac{b_{11}}{a+\varepsilon_{0}^{-1}}-\frac{\left(e_{44}-f_{12}\right)^{2}}{(\lambda+2 \mu)\left(a+\varepsilon_{0}^{-1}\right)} \geq 0 \\
h_{p}^{2}=\frac{b_{11}}{a+\varepsilon_{0}^{-1}} \geq \ell_{p}^{2} \geq 0
\end{gathered}
$$

Thus, we obtain two "microstructural"-related lengths $\left(\ell_{p}, \ell_{s}\right)$ and two "microinertial"-related lengths $\left(h_{p}, h_{s}\right)$. Note that the positiveness of the lengths stems from the assumed convexity of the energy density. Gradient dielectricity also yields the internal lengths $\left(\ell_{p}, \ell_{s}\right)$ and $\left(h_{p}, h_{s}\right)$ [46], while flexoelectricity leads to higher microstructural lengths, compared to gradient dielectricity. Typical material constants for PMMA were estimated and are given in Table 2 and are obtained from Refs. [9,48-53]. Note that the mechanical response is similar to the Mindlin's model of linear elastic solids with microstructure [54].

The material constants of Table 2 suggest microstructural lengths $\ell_{p}=2.9 \mathrm{~nm}$ and $\ell_{s}=4.521 \mathrm{~nm}$ and microinertial lengths $h_{p}=$ $3.2075 \mathrm{~nm}$ and $h_{s}=4.535 \mathrm{~nm}$ according to Eqs. (29) and (30), respectively.

The present work focuses on the mechanical response, solving Eqs. (24) for $\phi(\mathbf{x}, t)$ and (25) for $\mathbf{H}(\mathbf{x}, t)$. Once the displacement vector is found, the polarization vector can be found from the solution of Eq. (26) for $\chi(\mathbf{x}, t)$ and Eq. (27) for $\mathbf{K}(\mathbf{x}, t)$. We further note that polarization exhibits a size effect similar to the size effect of the mechanical displacement. Finally, electric potential can be obtained from the polarization vector from the solution of Eqs. (17) and (18). Note that, as in the classic elastodynamics, the present Helmholtz decomposition approach cannot be simply reduced to the static case, by omitting the acceleration terms [55].

Table 2 Typical material constants of PMMA

\begin{tabular}{lcc}
\hline \hline Constant & Value & Units \\
\hline$\mu\left(c_{44}\right)$ & 2.215 & $\mathrm{GPa}$ \\
$c_{11}$ & 9.585 & $\mathrm{GPa}$ \\
$/ e_{44}-f_{12} /$ & 7.015 & $\mathrm{Nm} / \mathrm{C}=\mathrm{V}$ \\
$/ e_{11}-f_{11} /$ & 56.12 & $\mathrm{Nm} / \mathrm{C}=\mathrm{V}$ \\
$b_{11}$ & $1.807 \times 10^{-6}$ & $\mathrm{Nm}^{4} / \mathrm{C}^{2}$ \\
$b_{44}$ (assumed) & $1.807 \times 10^{-6}$ & $\mathrm{Nm}^{4} / \mathrm{C}^{2}$ \\
$b_{77}$ (assumed) & $1.807 \times 10^{-6}$ & $\mathrm{Nm}^{4} / \mathrm{C}^{2}$ \\
$a$ & $6.275 \times 10^{10}$ & $\mathrm{Nm}^{2} / \mathrm{C}^{2}$ \\
$\epsilon_{0}$ & $8.85400 \times 10^{-12}$ & $\mathrm{C}^{2} /\left(\mathrm{Nm}^{2}\right)$ \\
$\rho$ & 1180 & $\mathrm{~kg} / \mathrm{m}^{3}$ \\
\hline \hline
\end{tabular}




\section{The Plane Strain/Plane Polarization Problem}

Section 2 is now particularized to the plain strain problem (parallel to the $x_{3}=0$ plane), where (in the absence of body forces and initial electric field) the displacement field is as follows:

$$
\begin{aligned}
& u_{1}\left(x_{1}, x_{2}, t\right)=\frac{\partial \phi}{\partial x_{1}}-\frac{\partial H_{3}}{\partial x_{2}} \\
& u_{2}\left(x_{1}, x_{2}, t\right)=\frac{\partial \phi}{\partial x_{2}}-\frac{\partial H_{3}}{\partial x_{1}} \\
& u_{3}\left(x_{1}, x_{2}, t\right)=0
\end{aligned}
$$

with unknown potentials $\phi\left(x_{1}, x_{2}, t\right)$ and $H_{3}\left(x_{1}, x_{2}, t\right)$.

Accordingly, the polarization vector is as follows:

$$
\begin{aligned}
& P_{1}\left(x_{1}, x_{2}, t\right)=\frac{\partial \chi}{\partial x_{1}}-\frac{\partial K_{3}}{\partial x_{2}} \\
& P_{2}\left(x_{1}, x_{2}, t\right)=\frac{\partial \chi}{\partial x_{2}}-\frac{\partial K_{3}}{\partial x_{1}} \\
& P_{3}\left(x_{1}, x_{2}, t\right)=0
\end{aligned}
$$

with unknown potentials $\chi\left(x_{1}, x_{2}, t\right)$ and $K_{3}\left(x_{1}, x_{2}, t\right)$.

The electric potential is $\Phi=\Phi\left(x_{1}, x_{2}, t\right)$.

The mechanical dynamic Eqs. (24) and (25) become

$$
\begin{gathered}
\nabla^{2} \phi-\ell_{p}^{2} \nabla^{4} \phi=\frac{1}{c_{p}^{2}}\left(\ddot{\phi}-h_{p}^{2} \nabla^{2} \ddot{\phi}\right) \\
\nabla^{2} H_{3}-\ell_{s}^{2} \nabla^{4} H_{3}=\frac{1}{c_{s}^{2}}\left(\ddot{H}_{3}-h_{s}^{2} \nabla^{2} \ddot{H}_{3}\right)
\end{gathered}
$$

The polarization equations (26) and (27) become

$$
\begin{gathered}
\nabla^{2} \chi-\ell_{p}^{2} \nabla^{4} \chi=\frac{1}{c_{p}^{2}}\left(\frac{e_{11}-f_{11}}{a+\varepsilon_{0}^{-1}} \nabla^{2} \ddot{\phi}\right) \\
\nabla^{2} K_{3}-\ell_{s}^{2} \nabla^{4} K_{3}=\frac{1}{c_{s}^{2}}\left(\frac{e_{44}-f_{44}}{a} \nabla^{2} \ddot{H}_{3}\right)
\end{gathered}
$$

In the above equations, $\nabla^{2}=\partial^{2} / \partial x_{1}^{2}+\partial^{2} / \partial x_{2}^{2}$ is the twodimensional Laplacian operator, and $\nabla^{4}=\nabla^{2} \nabla^{2}=\partial^{4} / \partial x_{1}^{4}+$ $2 \partial^{4} / \partial x_{1}^{2} \partial x_{1}^{2}+\partial^{4} / \partial x_{2}^{4}$ is the two-dimensional biharmonic operator. When all microstructural and microinertial lengths are zero, Eqs. (33) and (34) reduce to the plane stress classical elastodynamic equations [56].

\section{Uniformly Moving Rupture Tip}

A single moving rupture tip with constant rupture speed $V_{r}$ along the $x_{1}$ axis can be examined by making the standard steady-state coordinate transformation (simplifying the notation as, see Fig. 1):

$$
\xi=x_{1}+V_{r} t, \quad \eta=x_{2}
$$

The moving coordinate system $(\xi, \eta)$ is attached on the moving rupture tip.

Then, the equilibrium Eqs. (33) and (34) are reduced to

$$
\begin{aligned}
& \left(1-\frac{V_{r}^{2}}{c_{p}^{2}}\right) \frac{\partial^{2} \phi}{\partial \xi^{2}}+\frac{\partial^{2} \phi}{\partial \eta^{2}} \\
& \quad-\ell_{p}^{2}\left(\left(1-\frac{V_{r}^{2} h_{p}^{2}}{\ell_{p}^{2} c_{p}^{2}}\right) \frac{\partial^{4} \phi}{\partial \xi^{4}}+2\left(1-\frac{V_{r}^{2} h_{p}^{2}}{2 \ell_{p}^{2} c_{p}^{2}}\right) \frac{\partial^{4} \phi}{\partial \xi^{2} \partial \eta^{2}}+\frac{\partial^{4} \phi}{\partial \eta^{4}}\right) \\
& =0
\end{aligned}
$$

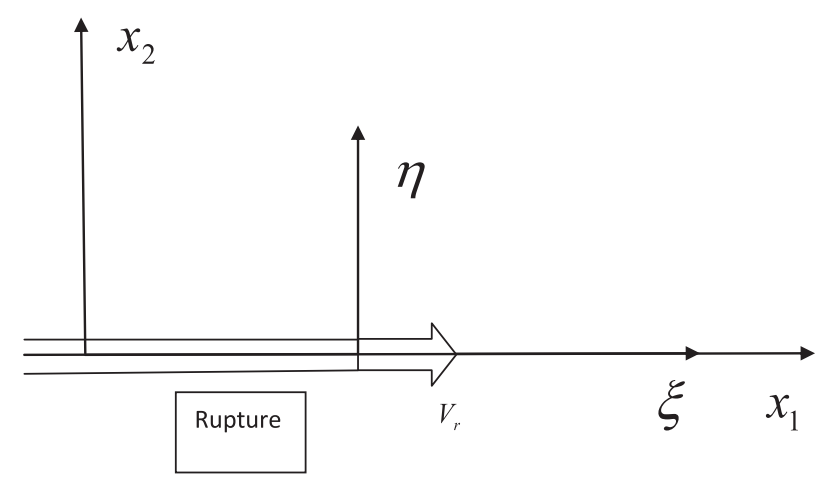

Fig. 1 The steady-state moving coordinate system $(\xi, \eta)$ with a rupture tip positioned at the center of the system. The crack tip is moving with the constant rupture speed $V_{r}$.

$$
\begin{aligned}
& \left(1-\frac{V_{r}^{2}}{c_{s}^{2}}\right) \frac{\partial^{2} H_{3}}{\partial \xi^{2}}+\frac{\partial^{2} H_{3}}{\partial \eta^{2}} \\
& \quad-\ell_{p}^{2}\left(\left(1-\frac{V_{r}^{2} h_{s}^{2}}{\ell_{s}^{2} c_{s}^{2}}\right) \frac{\partial^{4} H_{3}}{\partial \xi^{4}}+2\left(1-\frac{V_{r}^{2} h_{s}^{2}}{2 \ell_{s}^{2} c_{s}^{2}}\right) \frac{\partial^{4} H_{3}}{\partial \xi^{2} \partial \eta^{2}}+\frac{\partial^{4} H_{3}}{\partial \eta^{4}}\right) \\
& \quad=0
\end{aligned}
$$

Also, the polarization equations (35) and (36) become

$$
\begin{gathered}
\frac{\partial^{2} \chi}{\partial \xi^{2}}+\frac{\partial^{2} \chi}{\partial \eta^{2}}-\ell_{p}^{2}\left(\frac{\partial^{4} \chi}{\partial \xi^{4}}+2 \frac{\partial^{4} \chi}{\partial \xi^{2} \partial \eta^{2}}+\frac{\partial^{4} \chi}{\partial \eta^{4}}\right) \\
=\frac{V_{r}^{2}\left(e_{11}-f_{11}\right)}{c_{p}^{2}\left(a+\varepsilon_{0}^{-1}\right)}\left(\frac{\partial^{2} \phi}{\partial \xi^{2}}+\frac{\partial^{2} \phi}{\partial \eta^{2}}\right) \\
\frac{\partial^{2} K_{3}}{\partial \xi^{2}}+\frac{\partial^{2} K_{3}}{\partial \eta^{2}}-\ell_{p}^{2}\left(\frac{\partial^{4} K_{3}}{\partial \xi^{4}}+2 \frac{\partial^{4} K_{3}}{\partial \xi^{2} \partial \eta^{2}}+\frac{\partial^{4} K_{3}}{\partial \eta^{4}}\right) \\
=\frac{V_{r}^{2}\left(e_{11}-f_{11}\right)}{c_{p}^{2}\left(a+\varepsilon_{0}^{-1}\right)}\left(\frac{\partial^{2} H_{3}}{\partial \xi^{2}}+\frac{\partial^{2} H_{3}}{\partial \eta^{2}}\right)
\end{gathered}
$$

Equations (37) and (41) should be solved for $\chi(\xi, \eta)$ and $K_{3}(\xi, \eta)$, respectively, after solving Eqs. (38) and (39) with respect to $\phi(\xi, \eta)$ (dilatational waves) and $H_{3}(\xi, \eta)$ (shear waves).

In order to retain ellipticity of the equilibrium equations, we must have $h_{s}^{2} V_{r}^{2} /\left(\ell_{s}^{2} c_{s}^{2}\right)<1$ and $h_{p}^{2} V_{r}^{2} /\left(\ell_{p}^{2} c_{p}^{2}\right)<1$ [57,58]. Otherwise, the problem is hyperbolic and Mach lines are expected, even when the rupture tip motion is subsonic $\left(V_{r} / c_{s}<1\right.$ and/or $\left.V_{r} / c_{p}<1\right)$. Thus, the parameterization of the problem is synopsized in Fig. 2. For each type of wave (dilatational or shear), three regions appear: the elliptic (always subsonic), the hyperbolic, and the intermediate (always supersonic) region. The intermediate regions in the flexoelectric case are out of reach, since $h_{s} / \ell_{s} \geq 1$ and $h_{p} / \ell_{p} \geq 1$. The intermediate regions in the flexoelectric case are out of reach, since $h_{s} / \ell_{s} \geq 1$ and $h_{p} / \ell_{p} \geq 1$. Note, however, that although the elliptic region is also subsonic, the hyperbolic region is not necessarily supersonic.

\section{Pressure and Shear Results for the Hyperbolic Case}

In this section, we will examine the hyperbolic case, $h_{s}^{2} V_{r}^{2} /\left(\ell_{s}^{2} c_{s}^{2}\right) \geq 1$ and $h_{p}^{2} V_{r}^{2} /\left(\ell_{p}^{2} c_{p}^{2}\right) \geq 1$, together with the conditions $h_{s} / \ell_{s} \geq 1$ and $h_{p} / \ell_{p} \geq 1$, as indicated in Fig. 2 . Note, however, that this case cover both an intersonic rupture tip motion $\left(c_{p} / c_{s}>V_{r} / c_{s}>1\right)$ and a supersonic shear rupture tip motion. We will investigate the mechanical response as described by Eqs. (38) and (39).

Group theory may be handy at this point, selecting the spiral group (see, for example, Ref. [59] that accepts a "wave"-like 


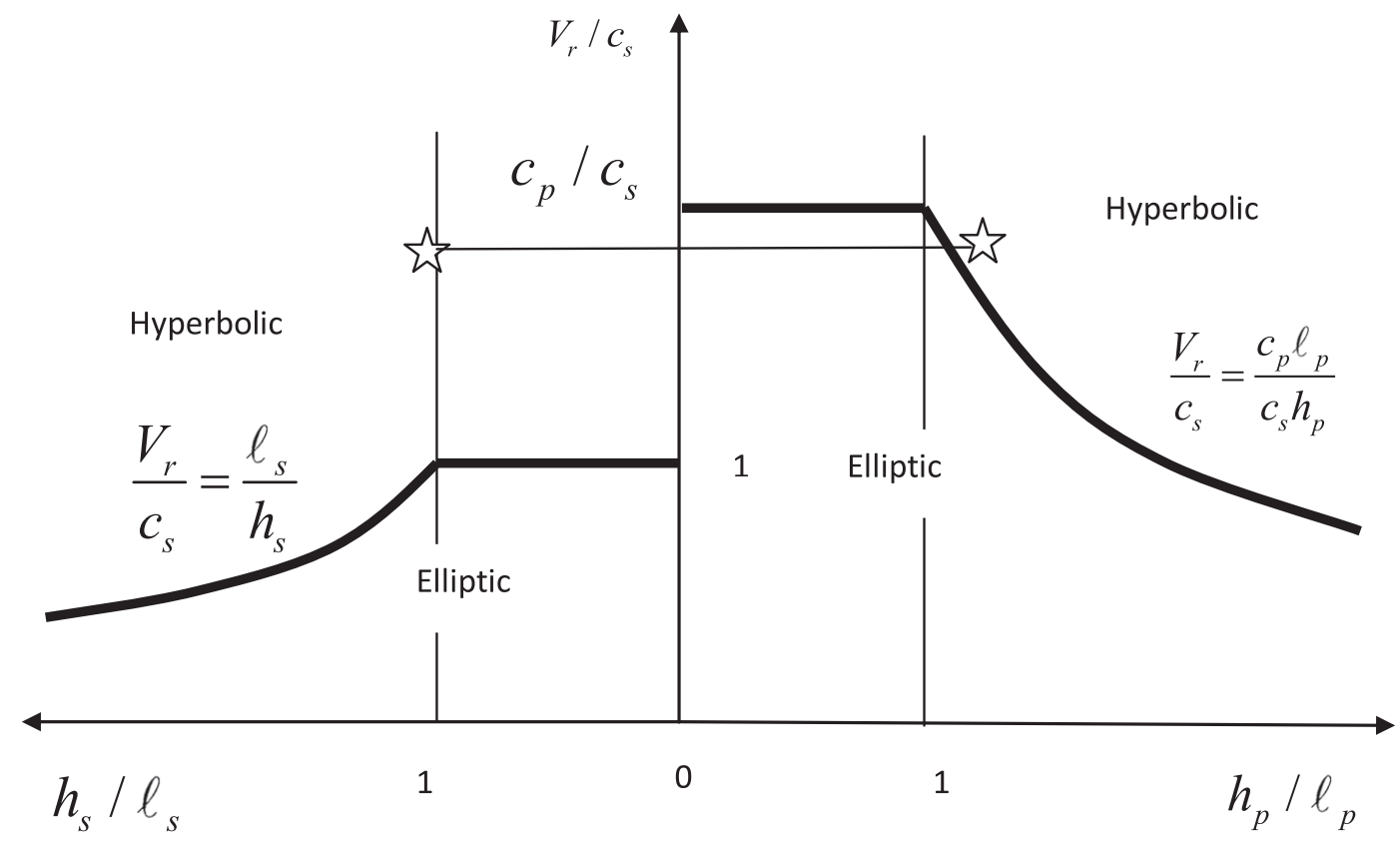

Fig. 2 Parameterization of the problem according to its three key parameters: $V / c_{s}$ and $c_{p} / c_{s}$ (as in the classical case), $h_{p} / \ell_{p}$ and $h_{s} / \ell_{s}$ (arise from the couple stress formulation and indicates dispersion effects). The particular example for PMMA [39] is also shown as a star.

coordinate). Invariance of the sequence of second and fourth derivatives with respect to the coordinates $\xi$ and $\eta$ leads to an exponential representation of the solution for the displacement. We first investigated the shear wave (39). If we seek solution with respect to a new variable:

$$
\bar{\eta}=\xi \pm \eta \sqrt{\frac{h_{s}^{2} V_{r}^{2}}{\ell_{s}^{2} c_{s}^{2}}-1}
$$

we obtain the invariance $\ln H_{3}=\bar{H}_{3}(\bar{\eta})$. Taking into account the partial differential Eq. (39), we find that

$$
\left[\frac{d^{2} \bar{H}_{3}}{d \bar{\eta}^{2}}+\left(\frac{d \bar{H}_{3}}{d \bar{\eta}}\right)^{2}\right]\left[\frac{h_{s}^{2} V_{r}^{2}}{\ell_{s}^{2} c_{s}^{2}}-\frac{V_{r}^{2}}{c_{s}^{2}}\right]=0
$$

Now, if $h_{s} / \ell_{s}=1$, Eq. (43) is true for any function $\bar{H}_{3}(\bar{\eta})$ and the problem reduces to the classic case. Otherwise,

$$
\bar{H}_{3}(\bar{\eta})=a_{i}+\ln \left[\bar{\eta}+b_{i}\right]
$$

where $a_{i}$ and $b_{i}$ are constants that can be adjusted to fit the boundary conditions along $\eta=0^{+}$.

Assuming $h_{s}^{2} V_{r}^{2} /\left(\ell_{s}^{2} c_{s}^{2}\right)>1$, Mach-like lines will develop, emanating from the rupture tip, making an angle $\beta_{s}$ with the $\xi$ line, as shown in Fig. 3:

$$
\sin \beta_{s}=\frac{\ell_{s}}{h_{s}} \frac{c_{s}}{V_{r}}
$$

The classic supersonic shear solution predicts $\sin \beta_{s}^{\text {classic }}=c_{s} / V_{r}$ and so $\beta_{s} \leq \beta_{s}^{\text {classic }}$. Therefore, Mach cones can appear for subsonic cases, as long as $h_{s}^{2} V_{r}^{2} /\left(\ell_{s}^{2} c_{s}^{2}\right)>1$, with slopes that are smaller than the classic ones. Note that as we approach the elliptic region, $h_{s}^{2} V_{r}^{2} /\left(\ell_{s}^{2} c_{s}^{2}\right) \rightarrow 1$, the angle $\beta_{s} \rightarrow \pi / 2$, which is the limit case of the elliptic region.

Now, let us investigate the dilatation wave (38). If we seek solution with respect to a new variable:

$$
\bar{\eta}=\xi \pm \eta \sqrt{\frac{h_{p}^{2} V_{r}^{2}}{\ell_{p}^{2} c_{p}^{2}}-1}
$$

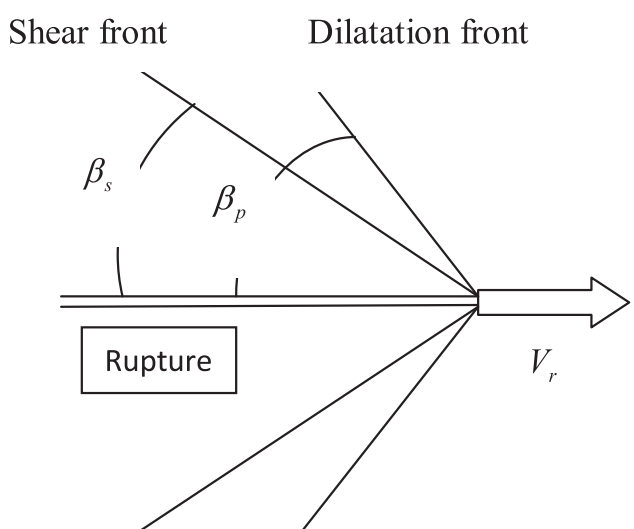

Fig. 3 Solution for the supershear $\left(V_{r} / c_{s}>1\right)$, but subsonic $\left(V_{r} / c_{p}\right.$ $<1)$, rupture motion in the hyperbolic regime $h_{p}^{2} V_{r}^{2} /\left(\ell_{p}^{2} c_{p}^{2}\right)>1$. Two sets of characteristic lines (Mach cone pairs) appear at angles $\sin \beta_{s}=\ell_{s} c_{s} /\left(h_{s} V_{r}\right)$ and $\sin \beta_{p}=\ell_{p} c_{p} /\left(h_{p} V_{r}\right)$.

we obtain the invariance $\ln \phi=\bar{\varphi}(\bar{\eta})$. Taking into account the partial differential equation (38), we find that

$$
\left[\frac{d^{2} \bar{\varphi}}{d \bar{\eta}^{2}}+\left(\frac{d \bar{\varphi}}{d \bar{\eta}}\right)^{2}\right]\left[\frac{h_{p}^{2} V_{r}^{2}}{\ell_{p}^{2} c_{p}^{2}}-\frac{V_{r}^{2}}{c_{p}^{2}}\right]=0
$$

Now, if $h_{p} / \ell_{p}=1$, Eq. (47) is true for any function $\bar{\varphi}(\bar{\eta})$, and the problem reduces to the classic case. Otherwise,

$$
\bar{\varphi}(\bar{\eta})=a_{i}+\ln \left[\bar{\eta}+b_{i}\right]
$$

where $a_{i}$ and $b_{i}$ are constants that can be adjusted to fit the boundary conditions along $\eta=0^{+}$.

Assuming $h_{p}^{2} V_{r}^{2} /\left(\ell_{p}^{2} c_{p}^{2}\right)>1$, additional Mach-like lines will develop (even if $V_{r}<c_{p}$ ). They are emanating from the rupture tip, making an angle $\beta_{p}$ with the $\xi$ line, as shown in Fig. 3:

$$
\sin \beta_{p}=\frac{\ell_{p}}{h_{p}} \frac{c_{p}}{V_{r}}
$$


The classic supersonic dilatation solution predicts $\sin \beta_{p}^{\text {classic }}=$ $c_{p} / V_{r}$ and so $\beta_{p} \leq \beta_{p}^{\text {classic }}$. Therefore, Mach cones can appear for subsonic cases, as long as $h_{p}^{2} V_{r}^{2} /\left(\ell_{p}^{2} c_{p}^{2}\right)>1$, with slopes that are smaller than the classic ones. Note that as we approach the elliptic region, $h_{p}^{2} V_{r}^{2} /\left(\ell_{p}^{2} c_{p}^{2}\right) \rightarrow 1$, the angle $\beta_{p} \rightarrow \pi / 2$, which is the limit case of this regime.

\section{Explaining an Experimental Paradox}

The recent experiments of Gori et al. investigated the dynamic growth of ultra-fast, shear, ruptures propagating along the interface of two PMMA plates held together, in frictional contact, under remotely applied compression and shear loads. The material of the plates was PMMA, a strongly flexoelectric polymer. These experiments were designed to mimic earthquake rupture growth along, strike-slip, geological faults. They represent the latest version of a class of dynamic rupture investigations, which were first introduced in the late nineties by Rosakis and his coworkers under the name of "laboratory earthquakes." The earlier versions of the "laboratory earthquake" setup involved the use of dynamic photoelasticity (a technique-sensitive shear but insensitive to dilatational deformation fields), and the experiments were conducted using plates of a photoelastic polymer, Hommalate-100. The latest experiments of Gori et al. utilized a new material, PMMA, as well as high-speed DIC, a diagnostic technique, capable of recording full-field maps of all components of displacement, strain, and strain rate near the propagating rupture in real time and at framing rates in excess of 1 milion frames per second $[40,41]$. The fortuitous use of a new (strongly flexoelectric) material, whose flexoelectric behavior has been studied independently as well as a more advanced diagnostic method capable of visualizing both shear and dilatational discontinuities, has resulted in the unexpected observation of dual sets (both shear and dilatational) of Mach lines emitted from the rupture tips that were propagating close, but well below, the dynamic dilatational wave speed of PMMA. The dual set of Mach lines, which were revealed in the PMMA experiments, is conceptually very similar to that schematically shown in Fig. 3 on the basis of our theoretical analysis.

In these experiments, $\beta_{p}=86 \mathrm{deg}$ and $\beta_{s}=32 \mathrm{deg}$. The rupture speed, which was independently measured from the rupture length versus time record, was found to be rather constant and was given by $V_{r} / c_{s}=1.92$ (while $c_{p} / c_{s}=2.08$ ) and was super shear but clearly subsonic. Obviously, in such a case, classical elastodynamics predicts only one set of Mach lines associated with jumps in shear strains and cannot explain the existence of the second set of dilatation-type Mach lines for such a supershear but clearly subsonic rupture. The present theory, however, provides an explanation of such a behavior. Indeed, if one used Eqs. (45) and (49) for estimating the angles of the shear and the dilatational Mach line pairs, respectively, one can retrieve both of the measured angles by simply inputting the measured normalized rupture speed and by assuming the micro length ratios to be equal to of $h_{p} / \ell_{p}=1.09$ and $h_{s} / \ell_{s}=1.0$, respectively. Indeed, this seems to be a very good prediction given the fact that the widely accepted electromechanical properties for PMMA from the open literature, listed in Table 2, predict $h_{p} / \ell_{p}=$ 1.11 and $h_{s} / \ell_{s}=1.00$.

It should also be noted that once the microscale ratios are estimated by the measured rupture speed and Mach line angles, one can determine and mark the experimental conditions in the context of Fig. 2 where regions of ellipticity and hyperbolicity are identified. On the one hand, the PMMA experiments (identified here with stars) clearly lie in regions where the governing equations, for both the shear and the dilatational potentials, are hyperbolic (regions where characteristics exist), and thus, the present theory accurately predicts the existence of dual sets of Mach lines. On the other hand, it is clear from that figure that if one considers only the position of the star relative to the vertical axis, classical elastodynamics would predict the development of only one pair of, shear, Mach lines and would be inconsistent with the experiments

\section{Conclusions}

In this work, we have examined the dynamic flexoelectric problem and solved it for ruptures moving with the constant speed. The flexoelectric material was stated as a dielectric solid that incorporates gradients of electric polarization and flexoelectricity due to strain gradients. The coupling of the mechanical with the electrical problem was condensed in a single mechanical problem with the displacement as prime unknown. The displacement was dissociated into the dilatation-related and the shear-related parts. In doing so, for each displacement part, two naturally emerging lengths (a microstructural and a microinertial one) appear explicitly in the problem. These four lengths are due to the interplay of the shear modulus, the dielectric constants, and the flexoelectric parameters.

Turning to the hyperbolic conditions, our analysis suggests that the slope of the Mach cones also depend on the microstructural as well as the microinertial lengths. Indeed, the slope of the Mach cones is smaller compared to that predicted by classical elasticity. Due to the existence of higher partial derivatives in the governing equations, hyperbolicity may occur at rupture speeds below one or both of the characteristic speeds (subsonic motion), and this is due to the existence of two microinertial lengths that must be higher than the two microstructural lengths.

The analysis indicated that the problem depends on four parameters: the two ratio of the rupture speed to the two wave speeds as in the classical elastodynamic (nondispersive) case and the two ratios of the microinertia to the microstructural lengths (one ratio for shear and one for dilatation), which introduces dispersion characteristics that are not present in the classical case. These parameters were found to break up the supersonic and the subsonic regions introducing intermediate regions inaccessible by classical elastodynamics.

By using our theoretical results, we are able to explain recent, unexpected, experimental observations of "intersonic" frictional rupture growth in PMMA (a strong flexoelectric solid). In these experiments, which were designed to mimic the earthquake rupture growth along frictional faults, both shear and dilatational Mach fronts were observed to form at the tips of dynamic frictional ruptures propagating at speeds above the shear wave speed but below the dilatational wave speed of the surrounding solid. Furthermore, for these experiments, we are able to predict the measured inclination angles of both the shear and the dilatational Mach lines by using independently measured values of the microstructural and microinertial lengths, available in the open literature.

The results are important for understanding the dynamic deformation and fracture/rupture of all dielectrics such as some geomaterials, ceramics, ice, perovskites, and polymers that exhibit strong flexoelectric effect, often uncoupled from piezoelectricity (centrosymmetric materials). Their success in predicting measure behavior in dynamic frictional rupture experiments (Laboratory Earthquakes) also highlights their possible relevance to earthquake source mechanics and earthquake seismology.

Finally, the results also apply to fast rupture that may be modeled in the context of couple stress elasticity. In such cases, the origin of the microstructural and microinertial lengths is very different than that the one proposed in this work. Indeed, atomistic calculations have managed to evaluate the strain gradient elastic tensors. However, little work has been done regarding the calculations of the microinertial lengths, and much remains to be done in this direction. Transient dynamic analysis is necessary to unveil the details of the dispersive nature of the dynamic problem.

\section{Appendix: The Completeness of the Helmholtz-Type Decomposition}

Following Sternberg [55], we give a short proof (Duhamel-type) for the completeness of the decomposition (22) and (23) that leads 
to Eqs. (24)-(27). We start from the Navier-type of equations (20) and (21), together with Eq. (16). Take the gradient on Eqs. (21) and (16) and eliminate the electric potential $\Phi$ to obtain

$$
\begin{aligned}
& \left(e_{44}-f_{12}\right) \nabla \cdot \nabla^{2} \mathbf{u}+\left(e_{12}+e_{44}-2 f_{44}\right) \nabla^{2} \nabla \cdot \mathbf{u} \\
& \quad+\left(b_{44}+b_{77}\right) \nabla \cdot \nabla^{2} \mathbf{P}+\left(b_{12}+b_{44}-b_{77}\right) \nabla^{2} \nabla \cdot \mathbf{P} \\
& \quad-\left(a+\varepsilon_{0}^{-1}\right) \nabla^{2} \nabla \cdot \mathbf{P}=0
\end{aligned}
$$

Take the gradient on Eq. (20) and replace the decomposition (22) and (23), with $\nabla \cdot \mathbf{u}=\nabla^{2} \phi$ and $\nabla \cdot \mathbf{P}=\nabla^{2} \chi$ and obtain

$$
\begin{gathered}
\left(e_{11}-f_{11}\right) \nabla^{4} \phi+b_{11} \nabla^{4} \chi-\left(a+\varepsilon_{0}^{-1}\right) \nabla^{2} \chi=0 \\
c_{11} \nabla^{4} \phi+\left(e_{11}-f_{11}\right) \nabla^{4} \chi=\rho \nabla^{2} \ddot{\phi}
\end{gathered}
$$

Note that the relations ()$_{11}=()_{12}+2()_{44}$ have been used for the material parameters.

Apply the Laplacian operator to Eqs. (A2) and (A3) and obtain

$$
\begin{gathered}
\left(e_{11}-f_{11}\right) \nabla^{6} \phi+b_{11} \nabla^{6} \chi-\left(a+\varepsilon_{0}^{-1}\right) \nabla^{4} \chi=0 \\
c_{11} \nabla^{6} \phi+\left(e_{11}-f_{11}\right) \nabla^{6} \chi=\rho \nabla^{4} \ddot{\phi}
\end{gathered}
$$

Eliminate $\chi$ from Eq. (A4), using Eq. (A5), we obtain

$-\frac{c_{11} b_{11}-\left(e_{11}-f_{11}\right)^{2}}{c_{11}\left(a+\varepsilon_{0}^{-1}\right)} \nabla^{6} \phi+\nabla^{4} \phi=\frac{\rho}{c_{11}} \nabla^{2} \ddot{\phi}-\frac{\rho}{c_{11}} \frac{b_{11}}{\left(a+\varepsilon_{0}^{-1}\right)} \nabla^{4} \ddot{\phi}$

Using Eq. (A4) with (A3), we obtain

$$
-\frac{c_{11} b_{11}-\left(e_{11}-f_{11}\right)^{2}}{c_{11}\left(a+\varepsilon_{0}^{-1}\right)} \nabla^{4} \chi+\nabla^{2} \chi=\frac{\rho}{c_{11}} \frac{\left(e_{11}-f_{11}\right)}{\left(a+\varepsilon_{0}^{-1}\right)} \nabla^{2} \ddot{\phi}
$$

Next, we apply curl operator on Eqs. (20) and (21) and apply the decomposition (22) and (23). Noting that $\nabla \times \mathbf{u}=-\nabla^{2} \mathbf{H}$ and $\nabla \times \mathbf{P}=-\nabla^{2} \mathbf{K}$, we obtain:

$$
\begin{gathered}
\left(e_{44}-f_{12}\right) \nabla^{4} \mathbf{H}+\left(b_{44}+b_{77}\right) \nabla^{4} \mathbf{K}-a \nabla^{2} \mathbf{K}=0 \\
c_{44} \nabla^{4} \mathbf{H}+\left(e_{44}-f_{12}\right) \nabla^{4} \mathbf{K}=\rho \nabla^{2} \ddot{\mathbf{H}}
\end{gathered}
$$

Take the Laplacian of Eqs. (A8) and (A9) and eliminate $\mathbf{K}$ to obtain:

$$
\begin{aligned}
- & \frac{c_{44}\left(b_{44}+b_{77}\right)-\left(e_{44}-f_{12}\right)^{2}}{c_{44} a} \nabla^{6} \mathbf{H}+\nabla^{4} \mathbf{H}=\frac{\rho}{c_{44}} \nabla^{2} \ddot{\mathbf{H}} \\
& -\frac{\rho}{c_{44}} \frac{\left(b_{44}+b_{77}\right)}{a} \nabla^{4} \ddot{\mathbf{H}}
\end{aligned}
$$

From (A8) and (A9), we obtain

$$
-\frac{c_{44}\left(b_{44}+b_{77}\right)-\left(e_{44}-f_{12}\right)^{2}}{c_{44} a} \nabla^{4} \mathbf{K}+\nabla^{2} \mathbf{K}=\frac{\rho}{c_{44}} \frac{\left(b_{44}-f_{12}\right)}{a} \nabla^{4} \ddot{\mathbf{H}}
$$

Finally, the electric potential $\Phi$ can be found from $\chi$ using

$$
-\varepsilon_{0} \nabla^{2} \Phi+\left(e_{11}-f_{11}\right) \nabla^{2} \chi=0
$$

Note that Eqs. (A6) and (A10) can be used for the static case, if $\rho=0$ (see Maraganti et al. [19]).

From Eqs. (A6) and (A10), we can obtain

$-\frac{c_{11} b_{11}-\left(e_{11}-f_{11}\right)^{2}}{c_{11}\left(a+\varepsilon_{0}^{-1}\right)} \nabla^{4} \phi+\nabla^{2} \phi-\frac{\rho}{c_{11}} \ddot{\phi}+\frac{\rho}{c_{11}} \frac{b_{11}}{\left(a+\varepsilon_{0}^{-1}\right)} \nabla^{2} \ddot{\phi}=\bar{a}$

$$
\begin{aligned}
&- \frac{c_{44}\left(b_{44}+b_{77}\right)-\left(e_{44}-f_{12}\right)^{2}}{c_{44} a} \nabla^{4} \mathbf{H}+\nabla^{2} \mathbf{H}-\frac{\rho}{c_{44}} \ddot{\mathbf{H}} \\
&+\frac{\rho}{c_{44}} \frac{\left(b_{44}+b_{77}\right)}{a} \nabla^{2} \ddot{\mathbf{H}}=\overline{\mathbf{b}}
\end{aligned}
$$

The new functions $\overline{\mathbf{a}}(\mathbf{x}, t)$ and $\overline{\mathbf{b}}(\mathbf{x}, t)$ are harmonic:

$$
\nabla^{2} \bar{a}=0 \nabla^{2} \overline{\mathbf{b}}=\mathbf{0}
$$

Define $\bar{A}(\mathbf{x}, t)$ and $\overline{\mathbf{B}}(\mathbf{x}, t)$ as follows:

$$
\begin{aligned}
\frac{\rho}{c_{11}} \ddot{\bar{A}}-\frac{\rho}{c_{11}} h_{p}^{2} \nabla^{2} \ddot{\bar{A}}=\bar{a} \\
\frac{\rho}{c_{44}} \ddot{\overline{\mathbf{B}}}-\frac{\rho}{c_{44}} h_{s}^{2} \nabla^{2} \ddot{\overline{\mathbf{B}}}=\overline{\mathbf{b}}
\end{aligned}
$$

which are also harmonic

$$
\nabla^{2} \bar{A}=0 \quad \nabla^{2} \overline{\mathbf{B}}=\mathbf{0} \quad \nabla \cdot \overline{\mathbf{B}}=0
$$

Define now the new functions $\phi_{1}(\mathbf{x}, t)=\phi+\bar{A}$ and $\mathbf{H}_{1}(\mathbf{x}, t)=\mathbf{H}+\overline{\mathbf{B}}$. It can be shown that

$-\frac{c_{11} b_{11}-\left(e_{11}-f_{11}\right)^{2}}{c_{11}\left(a+\varepsilon_{0}^{-1}\right)} \nabla^{4} \phi_{1}+\nabla^{2} \phi_{1}-\frac{\rho}{c_{11}} \ddot{\phi}_{1}+\frac{\rho}{c_{11}} \frac{b_{11}}{\left(a+\varepsilon_{0}^{-1}\right)} \nabla^{2} \ddot{\phi}_{1}=0$

$$
\begin{aligned}
& -\frac{c_{44}\left(b_{44}+b_{77}\right)-\left(e_{44}-f_{12}\right)^{2}}{c_{44} a} \nabla^{4} \mathbf{H}_{1}+\nabla^{2} \mathbf{H}_{1}-\frac{\rho}{c_{44}} \ddot{\mathbf{H}}_{1} \\
& +\frac{\rho}{c_{44}} \frac{\left(b_{44}+b_{77}\right)}{a} \nabla^{2} \ddot{\mathbf{H}}_{1}=\mathbf{0}
\end{aligned}
$$

Now, take $\mathbf{u}=\nabla \phi_{1}+\nabla \times \mathbf{H}_{1}+\mathbf{u}^{*}$ and $\mathbf{u}^{*}=-\nabla \bar{A}-\nabla \times \overline{\mathbf{B}}$. Using the identity $\nabla^{2} \overline{\mathbf{B}}=\nabla \nabla \cdot \overline{\mathbf{B}}-\nabla \times \nabla \times \overline{\mathbf{B}}$, we obtain

$$
\nabla \cdot \mathbf{u}^{*}=0, \quad \nabla \times \mathbf{u}^{*}=\overrightarrow{0}
$$

Then, there exist a (harmonic) function $\phi_{2}(\mathbf{x}, t)$ such that

$$
\mathbf{u}^{*}=\nabla \phi_{2}, \quad \nabla^{2} \phi_{2}=0
$$

Thus,

$$
\mathbf{u}=\nabla \phi_{1}+\nabla \phi_{2}+\nabla \times \mathbf{H}_{1}
$$

Inserting Eq. (A23) in the original equations (A20), (A21), and (A16) and taking $\mathbf{P}=\nabla \chi+\nabla \chi_{1}+\nabla \times \mathbf{K}$ with $\nabla^{2} \chi_{1}=0$ and $\nabla \cdot \mathbf{K}=0$, we obtain $\nabla \ddot{\phi}_{2}=\mathbf{0}$. Therefore, $\phi_{2}=\alpha(t)+t \beta(\mathbf{x})+g(\mathbf{x})$ with $\nabla^{2} \beta=0$ and $\nabla^{2} \gamma=0$. Define $\mathbf{u}=\nabla \phi_{3}+\nabla \times \mathbf{H}_{1}$ with $\nabla$. $\mathbf{H}_{1}(\vec{x}, t)=0$ and $\mathbf{P}=\nabla \chi_{2}+\nabla \times \mathbf{K}$ with $\chi_{2}=\chi+\chi_{1}$ and then

$$
\begin{gathered}
-\frac{c_{11} b_{11}-\left(e_{11}-f_{11}\right)^{2}}{c_{11}\left(a+\varepsilon_{0}^{-1}\right)} \nabla^{4} \chi_{2}+\nabla^{2} \chi_{2}=\frac{\rho}{c_{11}} \frac{\left(e_{11}-f_{11}\right)}{\left(a+\varepsilon_{0}^{-1}\right)} \nabla^{2} \ddot{\phi}_{3} \\
-\frac{c_{44}\left(b_{44}+b_{77}\right)-\left(e_{44}-f_{12}\right)^{2}}{c_{44} a} \nabla^{4} \mathbf{K}+\nabla^{2} \mathbf{K} \\
=\frac{\rho}{c_{44}} \frac{\left(b_{44}-f_{12}\right)}{a} \nabla^{4} \ddot{\mathbf{H}}_{1}
\end{gathered}
$$

This concludes the completeness theorem.

\section{Acknowledgment}

A. J. R. acknowledges support from the US Geological Survey (USGS) (Grant No. G20AP00037) and from the 2019 Caltech/ MCE Big Idea Fund (BIF). 


\section{References}

[1] Kogan, S. M., 1964, "Piezoelectric Effect During Inhomogeneous Deformation and Acoustic Scattering of Carriers in Crystals," Sov. Phys. Solid State, 5(10), pp. 2069-2070.

[2] Indebom, V. L., Loginov, E. B., and Osipov, M. A., 1981, "Flexoelectric Effect and Crystal Structure," Krystallografiya, 26(6), pp. 157-1162.

[3] Cross, L. E., 2006, "Flexoelectric Effects: Charge Separation in Insulating Solids Subjected to Elastic Strain Gradients," J. Mater. Sci., 41(1), pp. 53-63.

[4] Krichen, S., and Sharma, P., 2016, "Flexoelectricity: A Perspective on an Unusual Electromechanical Coupling," ASME J. Appl. Mech., 83(3), p. 030801

[5] Tagantsev, A. K., 1991, "Electric Polarization in Crystals and Its Response to Thermal and Elastic Perturbations," Phase Trans., 35(3-4), pp. 119-203.

[6] Zubko, P., Catalan, G., and Tagantsev, A. K., 2013, "Flexoelectric Effect in Solids," Annu. Rev. Mater. Res., 43(1), pp. 387-421.

[7] Dumitrica, T., Landis, C. M., and Yakobson, B. I., 2002, "Curvature Induced Polarization in Carbon Nanoshells," Chem. Phys. Lett., 360(1-2), pp. 182-188.

[8] Petrov, A. G., 2002, "Fleoelectricity of Model and Living Membranes," Biochim. Biophys. Acta., 1561(1), pp. 1-25.

[9] Chu, B., and Salem, D. R., 2012, "Flexoelectricity in Several Thermoplastic and Thermosetting Polymers," Appl. Phys. Lett., 101(10), p. 103905

[10] Ma, W., and Cross, L. E., 2003, "Strain-Gradient Induced Electric Polarization in Lead Zirconate Titanium Ceramics,” Appl. Phys. Lett., 82, pp. 3923-3925.

[11] Harado, J., Axe, J. D., and Shirane, G., 1971, "Neutron-Scattering Study of Soft Modes in Cubic $\mathrm{BaTiO}_{3}$," Phys. Rev. B, 4(1), pp. 155-162.

[12] Hikata, A. C., Elbaum, B., Chuck, B., and Truell, R., 1963, "Electrical-Charge Study in Sodium Chloride During Plastic Deformation,” J. Appl. Phys., 34(8), pp. 2154-2158.

[13] Linde, R. K., Murri, W. J., and Doran, D. G., 1966, "Shock-Induced Electrical Polarization of Alkali Halides," J. Appl. Phys., 37(7), pp. 2527-2532.

[14] Ahrens, T. J., 1966, "High-Pressure Electrical Behavior and Equations of State of Magnesium Oxide From Shock Wave Measurements,” J. Appl. Phys., 37(7), pp. 2532-2541.

[15] Weiner, J. H., 1981, "Hellmann-Feynman Theorem, Elastic Moduli and the Cauchy Relations," Phys. Rev. B, 24(2), pp. 845-848.

[16] Wert, C. A., 1986, "Internal Friction in Solids," J. Appl. Phys., 60(6), pp. 18881895 .

[17] Askar, A., Lee, P. C. Y., and Cakmak, A. S., 1970, "Lattice-Dynamics Approach to the Theory of Elastic Dielectrics With Polarization Gradient," Phys. Rev. B, 1(8), pp. 3525-3537.

[18] Maraganti, R., and Sharma, P., 2009, "Atomistic Determination of Flexoelectric Properties in Crystalline Dielectrics," Phys. Rev. B, 80(5), p. 054109.

[19] Maranganti, R., Sharma, N. D., and Sharma, P., 2006, "Electromechanical Coupling in Non-Piezoelectric Materials due to Nanoscale Nonlocal Size Effects: Green's Functions and Embedded Inclusions," Phys. Rev. B, 74(1), p. 014110. See also Erratum: Sharma, N. D., Landis, C., and Sharma, P., 2010, "Piezoelectric Thin-Film Super-Lattices Without Using Piezoelectric Materials," J. Appl. Phys., 108, p. 024304.

[20] Shen, S., and Hu, S., 2010, "A Theory of Flexoelectricity With Surface Effect for Elastic Dielectrics,” J. Mech. Phys. Solids, 58(5), pp. 665-677.

[21] Mindlin, R. D., 1968, "Polarization Gradient in Elastic Dielectric," Int. J. Solids Struct., 4(6), pp. 637-642.

[22] Sahin, E., and Dost, S., 1988, "A Strain-Gradients Theory of Elastic Dielectrics With Spatial Dispersion,” Int. J. Engng. Sci., 26(12), pp. 1231-1245.

[23] Hu, T., Yang, W., Liang, X., and Shen, S., 2017, "Wave Propagation in Flexoelectric Microstructured Solid," J. Elasticity, p. 10659

[24] Mao, S., and Purohit, P. K., 2015, “Defects in Fleoelectric Solids," J. Mech. Phys. Solids, 84, pp. $95-115$

[25] Brich, A. A., Tarasov, M. S., and Tsukerman, V. A., 1960, "Electrical Conductivity of Dielectrics in Strong Shock Waves," Soviet Phys. JETP, 11, pp. 15-17.

[26] Joigneau, S., and Thaivervin, J., 1958, "Electrical Conductivity of Sulfur Under the Action of a Shock Wave," C. R Acad. Sci., 246, pp. 3422-3425.

[27] Aki, K., and Richards, P. G., 1980, Qualitative Seismology. Volume II, W.H Freeman and Company, New York.

[28] Rosakis, A. J., Samudrala, O., and Coker, D., 1999, "Cracks Faster Than the Shear Wave Speed," Science, 284(5418), pp. 1334-1340.

[29] Rosakis, A. J., 2002, "Intersonic Shear Cracks and Fault Ruptures," Adv. Phys., 51(4), pp. 1189-1257.

[30] Xia, K., Rosakis, A. J., and Kanamori, H., 2004, "Laboratory Earthquakes: The Sub-Rayleigh-to-Supershear Rupture Transition,” Science, 303(5665), pp. 1859-1861.

[31] Rosakis, A. J., and Huang, Y., 2003, "Intersonic Debonding," Comprehensive Structural Integrity Handbook, Fracture of Materials From Nano to Macro, Volume Chap 8: Interfacial and Nanoscale Failure, W. Gerberich, and W. Yang, eds., Elsevier Ltd., New York, pp. 137-179.
[32] Rosakis, A. J., Lykotrafitis, G., Xia, K., and Kanamori, H., 2007, "Dynamic Shear Rupture in Frictional Interfaces: Speeds, Directionality and Modes," Treatise on Geophysics, 2nd ed., G. Schubert, ed., Vol. 4, Elsevier, Amsterdam, pp. 183-213.

[33] Rosakis, A. J., Rubino, V., and Lapusta, N., 2020, "Recent Milestones in Unravelling the Full-Field Structure of Dynamic Shear Cracks and Fault Ruptures in Real Time: From Photoelasticity to Ultrahigh-Speed Digital Image Correlation," J. Appl. Mech., 87(3).

[34] Bouchon, M., Bouin, M. P., Karabulut, H., Toksöz, M. N., Dietrich, M., and Rosakis, A. J., 2001, "How Fast Is Rupture During an Earthquake? NewInsights From the 1999 Turkey Earthquakes," Geophys. Res. Lett., 28(14), pp. 2723-2726.

[35] Ellsworth, W., Celebi, M., Evans, J., Jensen, E., Kayen, R., Metz, M., Nyman, D., Roddick, J., Spudich, P., and Stephens, C., 2004, "Near-Field Ground Motion of the 2002 Denali Fault, Alaska, Earthquake Recorded at Pump Station 10," Earthq. Spectra, 20(3), pp. 597-615.

[36] Song, S. G., Beroza, G. C., and Segall, P., 2008, "A Unified Source Model for the 1906 San Francisco Earthquake,” Bull. Seismol. Soc. Am., 98(2), pp. 823831

[37] Bao, H., Ampuero, J.-P., Meng, L., Fielding, E. J., Liang, C., Milliner, C. W., Feng, T., and Huang, H., 2019, "Early and Persistent Supershear Rupture of the 2018 Magnitude 7.5 Palu Earthquake," Nat. Geosci., 12(3), pp. 200-205.

[38] Amlani, F., Bhat, H. S., Simons, W. J. F., Schubnel, A., Vigny, C., Rosakis, A. J., Efendi, J., Elbanna, A., and Abidin, H. Z., 2020, "Supershear Tsunamis: Insights From the Mw 7.5 Palu Earthquake," Nat. Geosci. (submitted).

[39] Gori, M., Rubino, V., Rosakis, A. J., and Lapousta, N., 2018, "Pressure Shock Fronts Formed by Ultra-Fast Shear Cracks in Viscoelastic Materials," Nat. Commun., 9(1), p. 4754.

[40] Rubino, V., Rosakis, A., and Lapusta, N., 2019, "Full-Field Ultrahigh-Speed Quantification of Dynamic Shear Ruptures Using Digital Image Correlation," Exp. Mech., 59(551-582), pp. 1-32.

[41] Rubino, V., Rosakis, A. J., and Lapusta, N., 2020, "Spatiotemporal Properties of Sub-Rayleigh and Supershear Ruptures Inferred From Full-Field Dynamic Imaging of Laboratory Experiments," J. Geophys. Res. Solid Earth, 125, p. e2019JB018922.

[42] Giannakopoulos, A. E., and Th, Z., 2019, "Uniformly Moving Screw Dislocation in Flexoelectric Materials," Eur. J. Solids Mech. A Solids., 78, p. 103843.

[43] Maraganti, R., and Sharma, P., 2007, "Length Scales at Which Classical Elasticity Breaks Down for Various Materials," Phys. Rev. Letters, 98(19), p. 195504.

[44] Shu, L., Wei, X., Pang, T., Yao, X., and Wang, C., 2011, "Symmetry of Flexoelectric Coefficients in Crystalline Medium,” J. Appl. Phys., 110(10), p. 104106.

[45] Toupin, R. A., 1956, “The Elastic Dielectric,” J. Ration. Mech. Anal., 5, pp. 849 915.

[46] Mindlin, R. D., 1969, "Continuum and Lattice Theories of Influence of Electromechanical Coupling on Capacitance of Thin Dielectric Films," Int. J. Solids Structures, 5(11), pp. 1197-1208.

[47] Jackson, J. D., 1975, Classical Electrodynamics, John Willey \& Sons, New York.

[48] Mead, D. J., and Fuoss, R. M., 1942, "Electrical Properties of Solids. XIII Polymethyl Acrylate, Polymethyl Methacrylate, Polymethyl-a-Chloracrylate and Polychloroethyl Methacrylate," J. Am. Chem. Soc., 64(10), pp. 2380-2393.

[49] Harting, C., Kleppinger, R., and Jungnickel, B.-J., 1995, "Kerr-Effect Measurements on Poly(Vinylidine Fluoride) Poly(Methyl Methacrylate) and Their Blends," Polymer, 36(24), pp. 4553-4559.

[50] Hartmann, L., Gorbatschow, W., Hauwede, J., and Kremer, F., 2002, "Molecular Dynamics in Thin Films of Isotactic Poly(Methyl Methacrylate)," Eur. Phys. J., E8, pp. 145-154

[51] Kornyshev, A. A., and Vorotyntsev, M. A., 1981, "The Effect of Spatial Dispersion of the Dielectric Permittivity on the Capacitance of Thin Insulating Films: Non-Linear Dependence of the Inverse Capacitance on Film Thickness," Thin Solid Films, 75(2), pp. 105-118.

[52] Furukawa, T., Uematsu, Y., Asakawa, K., and Wada, Y., 1968, "Piezoelectricity, Pyroelectricity and Thermoelectricity of Polymer Films," J. Appl. Polym. Sci., 12(12), pp. 2675-2689.

[53] Han, C.-S., 2010, "Influence of the Molecular Structure on Indentation Size Effect in Polymers," Mater. Sci. Eng., A, 527(3), pp. 619-624.

[54] Mindlin, R. D., 1963, "Micro-Structure in Linear Elasticity," Arch. Rational Mech. Anal., 16(1), pp. 51-78.

[55] Sternberg, E., 1960, "On the Integration of the Equations of Motion in the Classical Theory of Elasticity," Arch. Rat. Mech. Anal., 6(1), pp. 34-50.

[56] Freund, 1. B., 1998, Dynamic Fracture Mechanics, Cambridge University Press, Cambridge, UK.

[57] Courant, R., and Hilbert, D., 1953, Methods of Mathematical Physics, Vol. 2, Intescience Publishers, New York.

[58] Renardy, M., and Rogers, R. C., 2004, An Introduction to Partial Differential Equations, 2nd ed., Springer Verlag, Heidelberg, Germany.

[59] Ames, K. A., and Ames, W. F., 1982, "On Group Analysis of the von Karman Equations,” Nonlinear Anal. Theory Appl., 6(8), pp. 845-853. 